\title{
Philosophiques
}

\section{Sur la phénoménologie évaluative et le caractère approprié des émotions}

\section{Stéphane Lemaire}

Volume 45, numéro 2, automne 2018

URI : https://id.erudit.org/iderudit/1055275ar

DOI : https://doi.org/10.7202/1055275ar

Aller au sommaire du numéro

Éditeur(s)

Société de philosophie du Québec

ISSN

0316-2923 (imprimé)

1492-1391 (numérique)

Découvrir la revue

Citer ce document

Lemaire, S. (2018). Sur la phénoménologie évaluative et le caractère approprié des émotions. Philosophiques, 45(2), 489-498. https://doi.org/10.7202/1055275ar d'utilisation que vous pouvez consulter en ligne.

https://apropos.erudit.org/fr/usagers/politique-dutilisation/ 


\title{
Sur la phénoménologie évaluative et le caractère approprié des émotions
}

\author{
STÉPHANE LEMAIRE \\ Université de Rennes
}

Le livre de Christine Tappolet Emotions, Values, and Agency offre une présentation détaillée et une défense systématique de la théorie perceptuelle des émotions et de certaines de ses implications. C'est le résultat d'un travail de vingt ans qui reprend et prolonge son livre, Émotions et valeurs, paru en 2000, aux Presses universitaires de France. On y retrouve un style à la fois simple, précis et fluide. Le livre nous conduit aisément à partir de thèses clairement dessinées vers des analyses riches et subtiles. C'est un excellent ouvrage, qui propose une position très cohérente et certainement attirante sur la nature des émotions et leurs liens avec les valeurs et les raisons.

La thèse centrale du livre est que les émotions sont des perceptions de valeur, ou plus exactement des expériences perceptuelles qui représentent des objets, événements ou états de choses comme ayant des propriétés de valeur. Plus précisément encore, ces expériences perceptuelles de valeurs ont un contenu non conceptuel. Ainsi, nous voyons le caractère effrayant du lion ou dégoûtant du cadavre, et cela ne nécessite pas qu'on dispose des concepts d'effrayant ou de dégoûtant. Je ne ferai pas la liste des avantages de cette position. Ils sont nombreux et, à certains égards, il me semble que cette position est la plus proche d'une interprétation plausible du sens commun. Ne reproche-t-on pas en effet à celui qui n'est pas dégoûté par la corruption des corps matériels et politiques de ne pas voir que c'est dégoûtant?

Il me semble toutefois que cette conception des émotions les enrichit de traits que les émotions ne possèdent pas et que cela contribue à une conception erronée de la normativité des émotions. Je vais donc proposer deux lignes d'objections. La première concerne la nature des émotions ellesmêmes, la thèse selon laquelle il s'agit d'expériences perceptuelles de valeur. La seconde concerne l'évaluation des émotions comme pouvant être appropriée ou pas à leur objet. Selon Tappolet, ce caractère approprié doit être analysé en termes de correction. J'essaierai de montrer qu'on a des raisons de rejeter cette idée.

Ma première ligne d'objection concerne l'idée que les émotions représentent des valeurs. Reprenons, pour commencer, la question que nous nous sommes posée avec Jérôme Dokic (Dokic et Lemaire 20I3). Cette question était la suivante: comment l'émotion en tant qu'expérience perceptuelle de valeur représente-t-elle non conceptuellement une valeur? En effet, Chris- 
tine Tappolet insiste sur un certain nombre d'analogies entre les émotions et les expériences perceptuelles. Deux éléments sont cruciaux: en premier lieu, les émotions sont dotées d'une certaine phénoménologie au même titre que les perceptions. Il y a une expérience émotionnelle comme il y a une expérience perceptuelle. Et en second lieu, les émotions sont dotées d'un certain contenu qui, comme pour les perceptions, est un contenu non conceptuel ${ }^{1}$. Cependant, le livre ne dit jamais comment l'expérience émotionnelle nous fait voir, nous présente de façon non conceptuelle, ou encore fait apparaître la valeur qui fait partie du contenu de l'émotion. Or, si on veut maintenir que les émotions sont bien des perceptions d'objets comme dotés de valeurs, il nous faut comprendre de quelle façon cette valeur apparaît à la personne qui éprouve l'émotion. Pour prendre une analogie simple: si je perçois un ballon rouge, alors mon expérience me présente un objet qui a une forme sphérique et la couleur rouge. Si on ne nous dit pas de quelle façon la propriété évaluative nous est présentée dans l'expérience, on pourra se demander si l'émotion nous donne bien effectivement à percevoir une valeur.

Un premier point semble clair: Tappolet ne soutient pas que la représentation de la valeur se fait à travers la présentation de l'émotion comme correcte. Moyennant l'équivalence conceptuelle néo-sentimentaliste que Tappolet accepte entre «être admirable» et «être l'objet d'une admiration appropriée » (p. 85), il serait possible à la perceptualiste de défendre que lorsque nous avons une expérience de peur à l'égard d'un objet, alors nous percevons en outre le caractère approprié de notre émotion. Cowan a récemment défendu cette position. Ainsi, il écrit: «Lorsque je regarde une photo de Pluton prise par la sonde spatiale New Horizons, j'admire le caractère admirable de l'univers. Ce faisant, il me semble aussi que mon expérience se représente elle-même comme appropriée (fitting) à la nature de l'objet que je contemple»(20I6, p. 74, je souligne).

On peut toutefois se demander dans quelle mesure nous percevons effectivement le caractère approprié de notre réponse. Il est plausible que nous puissions juger ou croire que notre émotion est appropriée parce que nous n'avons pas à l'esprit d'élément qui pourrait justifier que notre émotion n'est pas appropriée. Mais cette absence de défaiteur est-elle perçue? En outre, même si elle était perçue, cela ne suffirait pas à dire que l'émotion est une perception de valeur. Il faudrait encore que cette expérience perceptuelle de l'absence de défaiteur soit une partie de l'émotion elle-même. Or rien ne le garantit.

Mais laissons cette position de côté, car ce n'est pas l'option qui a la faveur de Tappolet. Cette dernière préfère adopter l'idée selon laquelle les

1. Tappolet ne cherche pas à caractériser positivement ce qu'est un contenu non conceptuel. Elle reste neutre sur ce point et se contente de la thèse négative selon laquelle la possibilité d'avoir une expérience dotée d'un contenu non conceptuel ne requiert pas la possession de concepts. 
propriétés évaluatives perçues ne sont pas dépendantes de la réponse (ne sont pas response-dependent). Cette position est à mon sens fragile. D'une part, elle engendre une tension interne avec la position que défend Tappolet sur les concepts évaluatifs et, d'autre part, on ne voit pas comment on pourrait l'élaborer de façon à la rendre correcte.

Commençons par la tension interne. En premier lieu, on peut douter qu'on perçoive des propriétés qui ne seraient pas dépendantes de la réponse comme telle. Par exemple, perçoit-on la propriété d'être comique ou la propriété d'être un danger par contamination qui pourrait être la valeur perçue lorsqu'on éprouve du dégoût. Dans le premier cas, le fait que personne ne sache caractériser le comique semble un indice; dans le second cas, il semble que nous n'envisagions le danger par contamination comme cause du dégoût qu'en cherchant à expliquer en termes évolutionnaires la fonction du dégoût.

À cette première objection, Tappolet semble toutefois parer, car elle soutient que si les propriétés évaluatives perçues sont des propriétés qui ne dépendent pas de la réponse, nous les rangeons toutefois sous des concepts qui dépendent de la réponse, comme l'admirable, etc. Ainsi, Tappolet écrit: «Les concepts évaluatifs doivent être expliqués en termes d'émotions appropriées ou qui conviennent, et sont par conséquent dépendantes de la réponse. Mais les propriétés qu'elles désignent sont parfaitement objectives au même titre que, dans de nombreux cas, les propriétés non relationnelles» (p. 79). Toutefois, cette position engendre une tension importante, car le contenu évaluatif des perceptions que constituent les émotions doit permettre, dans de bonnes conditions, de justifier des jugements évaluatifs. On ne peut justifier un jugement conceptuel sur la base d'un contenu non conceptuel perçu que si le contenu non conceptuel est repris par les concepts utilisés dans le jugement. Dès lors, si les expériences perceptuelles que sont les émotions présentent non conceptuellement des propriétés qui ne dépendent pas de la réponse, il est impossible qu'ils justifient des jugements évaluatifs qui impliquent des concepts qui dépendent de la réponse.

En d'autres termes, si les jugements évaluatifs portent sur des propriétés évaluatives dont les concepts sont compris à travers l'analyse néosentimentaliste, alors, pour que les émotions puissent justifier de tels jugements, il faudrait que l'admiration soit elle-même une perception d'objets comme ayant la propriété de rendre l'admiration appropriée. Mais, comme on vient de le voir, Tappolet rejette la thèse défendue par Cowan et selon laquelle l'admiration est la perception d'un objet comme ayant cette propriété.

Comment peut-on dès lors justifier un jugement évaluatif usant d'un concept de propriété évaluative dépendant de la réponse? En accord avec l'idée que développe Tappolet et selon laquelle les conditions dans lesquelles l'émotion est correcte sont l'objet d'une discussion a posteriori, la seule option disponible est de dire que le caractère approprié de l'émotion n'est pas établi par l'émotion. Mais, adopter cette thèse comme semble le faire 
Tappolet, c'est renoncer à l'idée selon laquelle l'émotion présente de façon non conceptuelle une propriété évaluative du type admirable, effrayant, etc. Comme on le voit, l'idée que l'émotion est une perception de valeur ne joue plus aucun rôle. L'émotion de peur ne présente son objet ni comme dangereux ni comme effrayant ${ }^{2}$. Elle nous présente son objet comme un objet qui fait peur, à la suite de quoi nous jugeons par ailleurs que cette peur est correcte.

Ma seconde objection est qu'on ne voit pas comment on pourrait élaborer l'idée d'expérience perceptuelle de valeur ou de présentation perceptuelle de valeur. Je présente donc une suite d'arguments sur la phénoménologie émotionnelle. En premier lieu, si la phénoménologie émotionnelle est surtout une phénoménologie corporelle, on est en droit de se demander comment l'expérience d'un certain état du corps pourrait présenter une propriété évaluative. A priori, si l'expérience émotionnelle est dans une large mesure une expérience du corps, alors elle représente le corps comme étant dans tel ou tel état. Bref, on ne voit pas en quoi cette expérience corporelle pourrait aussi être une présentation de valeur. Et même si on essaie de prendre l'expérience corporelle comme un tout, alors il s'agit de l'expérience d'une attitude comportementale, d'une orientation du corps, pas d'une propriété évaluative.

Il semble que, pour éviter cette difficulté, Tappolet doive défendre qu'un autre aspect, ou d'autres aspects de l'expérience émotionnelle présentent la valeur. Mais quels aspects? La focalisation de l'attention, le flux de nos pensées, l'expérience d'une motivation, tous ces éléments ne semblent pas susceptibles de présenter une valeur.

En second lieu, supposons même qu'il y ait une présentation de valeur, comment cette valeur présentée pourrait-elle être attribuée à un objet, événement ou état de choses qu'on se représente par ailleurs visuellement? Supposons qu'une certaine valeur négative soit présentée à travers notre expérience corporelle, la valeur présentée alors devrait présenter une propriété du corps, pas une propriété de l'objet que l'on voit par ailleurs. Comment la valeur présentée sur le corps pourrait-elle être attribuée à un objet perçu visuellement et donc totalement indépendamment de notre corps?

En troisième lieu, supposons même que la valeur soit présentée non conceptuellement et qu'on puisse comprendre comment elle est attribuée à un objet vu, comment cette explication peut-elle être transposée au cas où la base cognitive de l'émotion est une pensée ou un jugement. Si j'ai peur que la centrale nucléaire voisine pollue l'eau que je bois, il se peut que l'émotion résulte simplement du jugement que je viens de former, dont le contenu est propositionnel. Mais alors, comme l'ont remarqué Deonna et Teroni (que je

2. Et on peut se demander alors si sa position ne se rapproche pas dangereusement de celle de Deonna et Teroni (2OI2) pour autant qu'elle maintiendrait que la peur est correcte lorsque son objet est effrayant. 
me permets de reprendre ici en vertu de la convergence avec les objections précédentes), comment une propriété présentée non conceptuellement peutelle être attribuée à un objet qu'on se représente conceptuellement? Il y a là une question générale: est-il possible d'avoir des états mentaux à contenus mixtes, et dans lesquels une propriété présentée non conceptuellement serait attribuée à un objet qui ne serait pas présenté non conceptuellement. À ma connaissance, il n'existe pas de combinaisons de contenus représentationnels de cette sorte. Certes, on peut envisager que la catégorisation d'un objet perçu sous un concept d'espèce naturelle constitue un partie du contenu de la perception, et même que cela fasse une différence phénoménologique dans l'expérience visuelle, comme le soutient Susanna Siegel (20I0). Mais l'inverse semble une idée bien plus difficile à élaborer.

Enfin, et pour en finir avec le problème de la présentation phénoménologique de la valeur, je voudrais faire une petite remarque sur les liens possibles entre la théorie perceptuelle des émotions et les théories de l'appraisal défendues par un certain nombre de psychologues, en particulier Lazarus (I99I) et Scherer (Scherer et al. 200I). En effet, on pourrait se demander dans quelle mesure cette théorie psychologique des émotions pourrait apporter de l'eau au moulin de la théorie perceptuelle. Tappolet présente les théories de l'appraisal comme des variantes des théories cognitives (parfois dites aussi jugementalistes) (p. I2). Toutefois, il y a une différence importante. Si les émotions sont des jugements évaluatifs comme certains l'ont soutenu (Solomon I976), alors leur contenu est conscient. Par contre, les théories de l'appraisal soutiennent que l'appraisal n'est pas conscient. De ce fait, on pourrait envisager une certaine compatibilité entre les théories de l'appraisal et la théorie perceptuelle, voire même une convergence. On pourrait en effet soutenir d'une part qu'il y a un appraisal inconscient qui attribue une valeur à un objet, mais en outre que cette évaluation est rendue manifeste dans une expérience perceptuelle de valeur, un peu comme la catégorisation d'un objet pourrait, selon Siegel, se manifester dans l'expérience de l'objet. Dans ce cas, l'hypothèse la plus plausible serait que l'expérience, par exemple visuelle, de l'objet intentionnel de l'émotion serait modifiée de telle sorte qu'on verrait au sens strict que l'objet possède une valeur.

Cela me semble une hypothèse intéressante. Elle comporte toutefois au moins deux inconvénients. Le premier est que si l'expérience de valeur est visuelle lorsque la base cognitive est visuelle, auditive lorsque la base cognitive est auditive, etc., cela ne résout pas le problème des cas où la base cognitive est une croyance, sauf à dire que, dans ce cas, nous formons un jugement évaluatif, ce qui revient à abandonner la thèse perceptuelle.

Le second est que l'on se rapproche d'une hypothèse très fortement intellectualiste sur la perception des valeurs dans laquelle celle-ci est totalement froide. En effet, puisque la perception de valeur est, par exemple, visuelle, aucun des composants qu'on attribue en général aux émotions (la 


\section{Philosophiques / Automne 2018}

réponse physiologique, le comportement facial, la motivation, etc.) n'est impliqué par la perception de valeur. Certes, on pourra soutenir que l'émotion est causée par cette perception évaluative, mais c'est une position que doit rejeter Tappolet puisqu'elle admet que les émotions possèdent des composantes physiologiques, comportementales, de valence, etc. Bref, si l'idée d'une perception de valeur est alors envisageable, l'émotion elle-même ne peut pas être cette perception de valeur.

J'en viens à ma deuxième ligne d'objection. Celle-ci concerne l'évaluation des émotions. Selon la position de Tappolet, les émotions sont susceptibles d'être correctes ou incorrectes. Cette thèse dérive du fait que les émotions sont des expériences perceptuelles de valeur. Bien sûr, si on admet les objections faites plus haut, il devient plus difficile de défendre que les émotions sont correctes. On pourrait toutefois arguer que nous avons une raison indépendante de soutenir que les émotions sont soumises à un standard de correction. Cette raison dériverait de l'intuition commune selon laquelle les émotions peuvent être appropriées ou inappropriées à leurs objets. Ainsi disons-nous ordinairement qu'avoir peur d'une petite araignée inoffensive est excessif, et que c'est une erreur de ne pas admirer Bach. On peut alors offrir un argument à la meilleure explication, comme le fait Tappolet: les émotions sont appropriées parce qu'elles sont correctes.

En désaccord sur ce point, je voudrais soutenir qu'une autre explication du caractère approprié peut être formulée en termes de raisons pragmatiques, c'est-à-dire de raisons pratiques prudentielles ou morales, et qu'au fond seule cette autre explication fait sens. Mon argument est le suivant: les jugements sur le caractère correct d'une émotion sont par nature destinés à diverger des jugements sur son caractère pragmatique. Or, lorsque ces deux critères divergent, il n'y a aucune raison de préférer la correction. Donc, même si les émotions étaient correctes, cette évaluation n'aurait aucun rôle à jouer.

Je présenterai cette idée à travers deux exemples qui mettent en tension l'évaluation par la correction et l'évaluation pragmatique d'émotions.

Selon Tappolet, lorsqu'un morceau de musique est admirable à un certain degré, alors il est correct de l'admirer avec une certaine intensité correspondante. Supposons toutefois que Stéphane admire ce morceau de musique populaire des balkans plus qu'il n'est correct de l'éprouver. Bien sûr, ces deux faits sont compatibles. Il s'ensuit seulement que Stéphane éprouve une admiration excessive, incorrecte, et qu'il serait plus correct qu'il éprouve moins d'admiration. Mais supposons en outre que l'admiration forte que Stéphane éprouve pour ce morceau contribue plus fortement à la qualité de sa vie à court terme et à long terme. Ne serait-il pas légitimé de reprendre l'objection de Railton (I986) contre les conceptions objectives de la valeur et de dire que c'est "intolérablement aliénant». Et même il pourrait continuer: "Pourquoi serait-il plus correct d'admirer mon morceau favori moins que je ne l'admire? Pourquoi devrais-je me priver de ce plaisir, de cette émo- 
tion si enthousiasmante?» Le défenseur des émotions correctes pourrait peut-être répondre que le morceau en question n'est pas si important dans l'histoire de la musique ou que sa composition est assez simple. Ce à quoi Stéphane pourrait rétorquer: "Oui, certes, peut-être, mais quel est le rapport avec le degré de mon admiration? Pourquoi ces deux choses devraientelles être liées? Je suis ainsi fait que c'est là ce que j'admire; peut-être suis-je particulièrement sensible à la simplicité. En quoi serait-il mieux — plus correct - que j'admire moins ce que j'admire?»

Considérons maintenant un exemple qui pourrait s'appliquer à un groupe et au cas du danger qui contribue souvent à justifier l'approche néosentimentaliste. Imaginons une petite communauté d'Indiens vivant en Amazonie dans des conditions extrêmement dures et dans un environnement qui comporte des dangers permanents incomparablement supérieurs à ceux auquels nous faisons face dans nos sociétés. Appelons-les les Yanomos. Si l'émotion de peur est appropriée uniquement par rapport au danger, alors les Yanomos devraient vivre dans une peur permanente. Mais bien sûr, il ne serait pas prudent qu'ils vivent dans cette peur permanente, cela les empêcherait de s'adonner à d'autres activités importantes qui supposent un certain oubli du danger. Il est normal, pour ainsi dire, que leur disposition à la peur s'adapte. Autrement dit, d'un point de vue prudentiel, on a intérêt à avoir peur du danger, mais parfois aussi à ne pas en avoir peur étant donné les coûts de la peur. Cela semble expliquer pourquoi il est prudent d'avoir peur du danger, mais aussi prudent de ne pas avoir peur de ce qui n'est pas dangereux ou de ce qui l'est faiblement. Or il est plausible que cet équilibre entre les raisons prudentielles d'avoir peur et les raisons prudentielles de ne pas avoir peur soit différent selon les contextes dans lesquels on se situe. Bref, la prudence conseille aux Yanomos de ne pas avoir peur de certains dangers, ou d'en avoir moins peur que des universitaires européens, même si ces dangers sont identiques. Une fois admis l'existence de deux standards: d'un côté la peur correcte, et de l'autre la peur qu'il est prudent que les Yanomos éprouvent face à ces mêmes dangers, on peut se demander quelles doivent être les dispositions à la peur des Yanomos. Certainement pas celles qui correspondent aux émotions correctes. Le seul critère pertinent semble être le critère prudentiel. Donc, une fois de plus, la correction (et en général tout critère de convenance non pragmatique) semble offrir un standard dépourvu de tout intérêt.

Je vois deux stratégies possibles pour parer à cette objection. Selon la première, le défenseur d'un standard de correction adopterait en réponse une conception relationnelle des valeurs. Après tout, c'est une thèse compatible avec une théorie perceptuelle des émotions, même si ce n'est pas la position de Tappolet qui reste objectiviste: les émotions donneraient à percevoir des valeurs relatives au sujet. Mais, de deux choses l'une. Ou bien ces émotions correctes pour un individu ou un groupe sont celles qui sont pragmatiquement justifiées, ou bien ce n'est pas le cas. Si elles ne le sont pas, 
alors nous pouvons de nouveau demander: Pourquoi? Pourquoi Stéphane devrait-il admirer moins son morceau de musique préféré, et pourquoi les Yanomos devraient-ils éprouver plus de peur qu'il est prudent d'en éprouver. Bref, pour éviter ces nouvelles objections, la seule solution est de rabattre les émotions correctes pour un individu ou un groupe sur celles qui sont pragmatiquement justifiées. Et, effectivement, c'est le but visé par la relativisation des valeurs. Cette stratégie pose toutefois un problème. Si on cherche à accorder la correction à la justification pragmatique, alors la correction ne joue, à nouveau, aucun rôle, et en réalité on pourrait s'en passer ${ }^{3}$. Au mieux, son maintien ne sert à rien d'autre qu'à redire en terme de correction ce qui est déjà établi par une analyse des valeurs affectives en termes d'émotions pragmatiquement justifiées.

Une deuxième stratégie de réponse est toutefois plus prometteuse. Elle consiste à montrer que, même si la normativité pragmatique doit toujours l'emporter, la correction a tout de même une pertinence. Pour cela, on s'appuiera sur une analogie avec les croyances. Certes, pourra-t-on dire, il est pragmatiquement préférable d'avoir dans certaines circonstances des croyances fausses, mais cela n'enlève rien au fait que ces croyances sont fausses et de ce fait incorrectes.

On peut donner deux interprétations de cette réponse. Selon une première interprétation, la réponse part de l'idée que les émotions ont des contenus évaluatifs et de ce fait sont analogues à des croyances ou des perceptions. Dans ce cas effectivement, on peut bien distinguer, comme pour les croyances, leur correction et leur avantage pragmatique, et la correction possède un intérêt propre, elle permet la connaissance. Le défaut de cette interprétation est toutefois qu'on ne peut plus dire alors qu'on a un argument indépendant et supplémentaire en faveur de l'idée que les émotions peuvent être correctes ou pas. De nouveau, tout repose sur la plausibilité de la thèse perceptuelle initiale. Si, par contre, on ne fait pas d'abord l'hypothèse que les émotions sont correctes par nature, alors la question cruciale est de savoir s'il existe vraiment une analogie et en quoi elle consiste. L'argument central du défenseur de l'analogie est de souligner que, même si une émotion est préférable du point de vue pratique, nous avons l'intuition qu'elle n'est pas forcément appropriée. C'est le fameux problème des raisons de la mauvaise sorte ${ }^{4}$. Si un démon nous menace des pires tortures afin que nous l'admirions, nous avons certainement une raison prudentielle de l'admirer, mais il ne semble pas que nous voudrions dire que le démon est d'autant plus admirable. Ce sont de tels exemples qui justifient une analogie avec les croyances. Il existerait donc bien une dualité de standard, comme pour

3. Le lecteur reconnaittra ici (paradoxalement) une adaptation de l'argument de Enoch contre le subjectivisme. Voir Enoch 2005.

4. Cf. pour ce problème les deux articles de références: D’Arms and Jacobson (2000), et Rabinowicz \& Rønnow-Rasmussen (2004). 
la croyance. On notera toutefois que cet argument ne montre pas qu'il existe un standard indépendant des raisons pragmatiques, mais que certaines considérations pragmatiques ne sont pas pertinentes.

Quoi qu'il en soit, et sans vouloir entrer complètement dans le très difficile débat qui entoure les raisons de la mauvaise sorte, je voudrais noter qu'il existe entre les émotions et les croyances une disanalogie importante qui semble s'opposer à l'idée que les émotions ont comme les croyances un double standard. En effet, nous avons la plupart du temps un intérêt pragmatique à posséder des croyances correctes, parce que ces croyances nous permettent de mieux nous guider et d'agir plus efficacement dans le monde. Mais qu'en est-il des émotions? Les émotions correctes ont-elles un intérêt instrumental autonome? Autrement dit, quel est l'avantage d'identifier ce qui est effrayant et dégoûtant? En ce qui concerne les Yanomos et la peur, ceux-ci n'ont aucun intérêt à avoir des émotions de peur correctes au sens de Tappolet. La seule chose qui compte est qu'ils aient des émotions de peur justifiées prudentiellement. Certes, répondra la défenseuse de l'analogie, mais cela tient au contexte spécifique des Yanomos. En réalité, et la plupart du temps, l'émotion correcte pointe vers le danger dont il faut tenir compte et avec l'intensité pertinente. Donc les émotions correctes sont utiles, comme les croyances. Mais le défenseur de la position pragmatiste pourra de nouveau objecter (comme plus haut par rapport à la relativisation) que si la correction ne fait que pointer (dans les meilleurs des cas) vers les émotions qu'il est par ailleurs justifié pratiquement d'avoir, alors l'hypothèse de la correction est superflue. On peut se contenter de la justification pragmatique des émotions. Bref, il y a une disanalogie importante entre croyances et émotions. Il est utile d'un point de vue pragmatique de former des croyances qui répondent à un standard autre que pratique — la correction - , mais ce n'est pas le cas pour ce qui est des émotions ${ }^{5}$.

\section{Bibliographie}

Cowan, Robert. "Epistemic Perceptualism and Neo-Sentimentalist Objections", Canadian Journal of Philosophy, 201 6, vol. 46, $\mathrm{n}^{\circ} \mathrm{I}, \mathrm{p} .59-8 \mathrm{I}$.

D’Arms, Justin, et Daniel Jacobson. "The Moralistic Fallacy: On the "Appropriateness" of Emotions" ", Philosophy and Phenomenological Research, vol. 6I, $\mathrm{n}^{\circ} \mathrm{I}, 2000$, p. 65-90.

Deonna, Julien, et Fabrice Teroni. The Emotions: A philosophical Introduction, Routledge, 20 I 2.

Dokic, Jérôme, et Stéphane Lemaire. "Are Emotions Perceptions of Value?», Canadian Journal of Philosophy, 2013, vol. 43, $\mathrm{n}^{\circ}$ 2, p. 227-247.

5 Je tiens à remercier énormément Christine Straehle qui en organisant un symposium sur le livre de Christine Tappolet m'a permis de tester différentes hypothèses sur les positions de cette dernière. Les discussions que j'ai pu avoir autour de ce symposium et en particulier avec Christine Tappolet m'ont permis d'y voir beaucoup plus clair, et de rendre mes arguments plus pertinents, du moins je l'espère. 
Enoch, David. "Why Idealize? ", Ethics, vol. I I 5, n 4, 2005, p. 759-787.

Lazarus, Richard S. Emotion and Adaptation, Oxford University Press, Oxford, I99I.

Rabinowicz, Wlodek, \& Toni Rønnow-Rasmussen. «The Strike of the Demon: On Fitting Pro-Attitudes and Value ", Ethics, vol. I I 4, n 3, 2004, p. 39I-423.

Railton, Peter. "Facts and Values", Philosophical Topics, vol. I4, $\mathrm{n}^{\circ}$ 2, I986, p. 5-3I.

Scherer, Klaus R., Angela Schorr et Tom Johnstone (eds.), Appraisal Processes in Emotion: Theory, Methods, Research, Oxford University Press, $200 \mathrm{I}$.

Siegel, Susanna. The Contents of Visual Experience, Oxford University Press, Oxford, 20II.

Solomon, Robert C. The Passions: Emotions and the Meaning of Life, Hackett Publishing, I976.

Tappolet, Christine. Émotions et valeurs, Presses universitaires de France, Paris 2000.

—. Emotions, Values, and Agency, Oxford University Press, Oxford, 2016. 